\title{
Research on D2D Communication
}

\author{
Fangni Chen ${ }^{1,}$ a , Zhongpeng Wang ${ }^{1, b}$ \\ ${ }^{1}$ Zhejiang University of Science and Technology, Hangzhou, China \\ ccfnini@163.com, ${ }^{b}$ wzp1966@sohu.com
}

\begin{abstract}
The significant development in mobile internet greatly enrich our daily life, meanwhile it challenges the capacity of the current cellular networks. The limited frequency resources are the main bottleneck of the development of cellular networks. Device-to-device(D2D) communication is a new technology that allows mobile terminals to directly communicate with each other by sharing the resources of cells under the control of cellular systems. D2D communication is introduced into cellular networks to improve resource utilization efficiency and increase system throughput. However, it also gives rise to many new challenges. We first introduce the system model and operating principle of D2D communication. Next a survey is made on the recent researches aiming to achieve D2D communication in cellular networks, including session set up, resource optimization, and application.
\end{abstract}

Keywords: Device-to-device, session set up, resource optimization, application.

\section{Introduction}

As the main network carrier of the mobile internet, tor short messages, but complex multi-media such as video and other kinds of data. These changes challenge the capacity of the current cellular networks. The limited frequency resources are the main bottleneck of the development of cellular networks. Increasing the spectrum efficiency is one of the most significant ways to improve the capacity of cellular networks. Techniques that can enable spatial reuse attract much attention in recent years, as they can greatly increase the spectrum efficiency without too many costs. Among them, the Device-to-Device (D2D) 
communication makes use of the proximity of two devices that need to communicate with each other, enables direct communication between them. The most attractive benefit of enabling D2D in cellular networks is that the D2D communication may reuse the radio resources of the cellular users so as to increase the spectrum efficiency. Therefore, D2D communication becomes one of the hot topics in cellular network research.

Device-to-device(D2D) communication is a new technology that allows mobile terminals to directly communicate with each other by sharing the resources of cells under the control of cellular systems. D2D communication can reduce the burden of base station[1-2], reduce the communication time delay[3]. Compared with the cellular communications, D2D communication only occupies half of the spectrum resources. In addition, the nearby users can reduce the transmission power by using D2D communication[4]. Therefore, D2D communication is expected to become the key technologies of the future mobile communication.

\section{System Model}

D2D can be deployed in a FDD (Frequency Division Duplexing) or TDD (Time Division Duplexing) cellular network mode. Although the TDD model can simplify the measurement of the channel requirements, it also has higher requirements of switching between uplink and downlink. Assuming a generic cellular network, the base station is sitting in the center of a cell. OFDM technology is deployed in the network. the spectrum resource is divided into a series of mutually orthogonal subcarriers, which are assigned to different users who may not interfere with each other by using orthogonal resources. Users are divided into two categories: 1) the traditional cellular users, who communicate through the base station; 2) D2D users, who use direct communication between each other. They can also be carried out in a cellular communication, and can realize the switching of the above two communication modes. As shown in Fig. 1, user 1 and user 2 use cellular communication mode, user 3 and user 4 use D2D mode communication mode. 

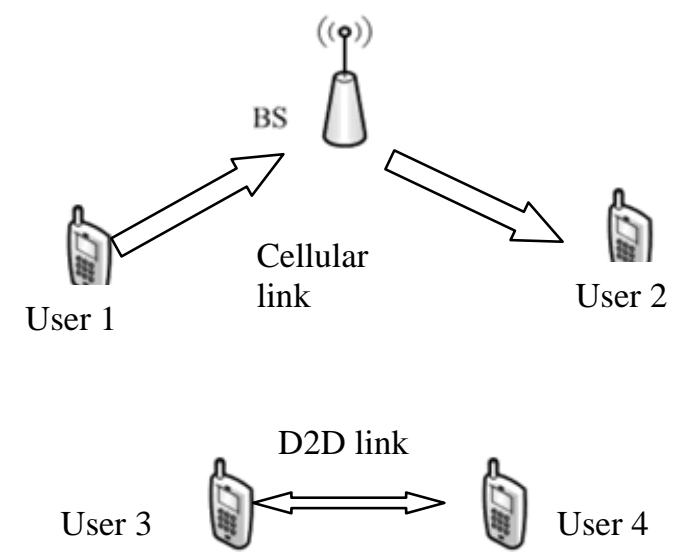

Fig. 1. System Model

The introduction of D2D communication technology in cellular system can not only improve the short distance communication experience for users, but also improve the spectrum efficiency of the system. Macro station only need to spend a certain mount of signaling to control the D2D communication, and does not need to be responsible for data transfer between both sides, thus reducing the load.

Similar end-to-end direct communication technology such as Bluetooth and WLAN ( Wireless Local Area Network), which are working in the unlicensed bands with complex interference environment. The reliability can not be guaranteed, and the connection establishment between the equipment needs manual pairing and the AP (Access Point) configuration. While the D2D technique uses cellular resources, and D2D connection can be controlled by the base station, so the interference between D2D and cellular system is easier to control. What's more, the D2D session establishment and the handover are transparent to the user, which are completely controlled by the base station. So the deployment of D2D in cellular networks can effectively reduce the load of base stations, reduce the battery consumption of terminals, improve the spectral efficiency. 


\section{Research contents}

In recent years, the main research areas of D2D communication include the D2D session set up, D2D mode selection, D2D power control, and D2D application research.

D2D Session Set up. The session setup is to establish a D2D link for data transmission in a cellular network. In fact, the terminal user must satisfy certain conditions (distance condition, or interference limitation, etc.) to realize D2D communication. When the condition is not satisfied, cellular communication is on. When it meets the D2D condition, how to choose the communication mode to enhance the system performance is also a key problem. K. Doppler [5] proposed that the macro base station controls the D2D beacon channel to assist the D2D found, thus avoiding D2D beacon monitoring over multiple channels, save the energy consumption. R Huang [6] proposed a D2D set up mechanism based on efficiency considerations. [7] proposed a set up method based on the mechanism of selective scanning and comparison of data rate. [8] proposed a set up mechanism using an autonomous selection of communication mode and reduce interference effectively.

Resource Optimization. Resource optimization of D2D communication is a key topic in the research of D2D. The significance of resource optimization is to guarantee user's QoS and improve resource utilization efficiency through the allocation and management of wireless resources. The main research contents include the mode selection, power control and channel allocation.

D2D technology can not only work in the special resources, but also can share the same system resources with other cellular user in the system. [9] proposed that D2D communication can work in three modes including dedicate model, multiplexing model and cellular model. Among them, dedicate mode is to allocate special resource for D2D, and will not bring additional interference to the system. Therefore, when the system has idle resources, dedicate resources can be allocated to D2D. However, it will reduce the system spectrum efficiency. Multiplexing mode is that the system makes the D2D to share other cellular resource block. This model can improve the efficiency of system resources, but it will bring new problems such as introducing the inter-cell interference between users. We need to 
ensure the normal cellular users, can not make the performance of cellular communication reduced to a certain level; at the same time, we also need to ensure good performance of D2D communication. In the cellular mode, D2D uses the traditional base station relay communication method. [10] discussed the model selection problem, proposed a mode selection method based on the optimization of the power.

Although the D2D communication is a direct end-to-end communication, the communication process is still under the control of the base station. In cellular mode and dedicate mode, each link uses the orthogonal resource in order not to interfere with each other. Users can use the maximum power in order to achieve the best performance. In multiplexing mode, the transmit power must ensure the QoS of D2D users, and not to introduce too much interference to cellular user who share the same frequency. Therefore, power control is very important in multiplexing mode. [11] analyzed the D2D communication performance under a simple power control algorithm. The authors gave the SINR distribution of D2D users and multiplexing cellular users. The results displayed in such power control strategy, D2D users can obtain reliable QoS under the guarantee of QoS of cellular users.

Channel allocation is a popular research direction for the D2D communication. [12] clearly pointed out the importance of channel assignment in D2D communication to the user performance. In fact, channel allocation is usually jointly designed with mode selection and power control algorithm. Research of this kind of thought is usually to improve the spectrum efficiency and energy efficiency, and the channel assignment problem is modeled as a programming problem. [13] gave the complete solution to the channel assignment problem for a scenario of D2D users reusing a cellular users resource. In the light of the lack of the centralized algorithm which requires a lot of channel state information, many studies devoted to the design of distributed channel assignment algorithm.

Application. Research on D2D application mainly consider how to combine D2D communication technology with the practical application, how to optimize the application through the D2D communication, and at the same time to optimize the D2D communication through the analysis of application scenarios. D2D communication provides the direct communication between the cellular terminal, 
which can provide more excellent and more efficient services for cellular terminals. The typical applications of D2D communication include multicast, video distribution, M2M and so on. [14] pointed out that communication with D2D as the relay technology can improve capacity and coverage. [15] considered D2D communication for multi hop network scenarios. In addition, P.Wang proposed application of D2D communication [16] in smart grid nodes.

\section{Summary}

D2D communication technology can improve the spectrum efficiency, reduce base load, reduce power consumption, improve the user experience and many other advantages. This paper launched a series of studies and analysis of D2D communication in session set up, resource optimization and application design. It may have certain reference significance and practical value in the future application of D2D communication in cellular network.

\section{References}

[1] P.Janis, C.H.Yu, K.Doppler, et al. Device-to-device communication underlaying cellular communications systems . International Journal of Communications, Network \& System Sciences, Vol. 2(3) (2009), p. 169-178.

[2] K.Doppler , M.Rinne , C.Wijting , et al . Device-to-device communication as an underlay to LTE-advanced networks . IEEE Communications Magazine, Vol. 47 (12) (2009), p. 42-49.

[3] B.Kaufman, B.Aazhang. Cellular networks with an overlaid device to device network. IEEE 42nd Asilomar Conferenceon Signals, Systems and Computers, (2008), p. 1537-1541. 
[4] G.Fodor, E.Dahlman, G.Mildh, et al. Design aspects of network assisted device-to-device communications. IEEE Communications Magazine, Vol. 50( 3) (2012), p. 170-177.

[5] K. Doppler, C. B. Ribeiro, J. Kneckt. Advances in D2D communications: Energy efficient service and device discovery radio, International Conference on Wireless Communication, Vehicular Technology, Information Theory and Aerospace and Electronic Systems Technology. (2011), p. 1-6.

[6] P. Huang, E. Qi, M. Park, A. Stephens. Energy efficient and scalable device-to-device discovery protocol with fast discovery, IEEE Communications Society Conference on Sensor, Mesh and Ad Hoc Communications and Networks. (2013), p. 1-9.

[7] N. Golrezaei, A. G. Dimakis, A. F. Molisch. Device-to-device collaboration through distributed storage, IEEE Global Communications Conference. (2012), p. 2397 - 2402.

[8] J. C. Li, M. Lei, F. Gao. Device-to-device (D2D) communication in MU-M1MO cellular networks, IEEE Global Communications Conference. (2012), p. 3583 - 3587.

[9] 3GPP. TR 22.803 v. 12.2.0. Feasibility study for proximity services (ProSe). (2013).

[10] C. Yu, O. Tirkkonen, K. Doppler, C_ Ribeiro. Power optimization of device-to-device communication underlaying cellular communication, IEEE International Conference on Communications. (2009), p. 1-5.

[11] C. Yu, O. Tirkkonen, K. Doppler, C. Ribeiro. On the performance of device-to-device underlay communication with simple power control, in IEEE Vehicular Technology Conference. (2009), p.1-5.

[12] H. Xing, S. Hakola. The investigation of power control schemes for a device-to-device communication integrated into OFDMA cellular system, in IEEE International Symposium on Personal Indoor and Mobile Radio Communications. (2010), p. 1775-1780. 
[13] D. Feng, L. Lu, Y. Wu, G. Y. Li, G. Feng, S. Li. Device-to-device communications underlaying cellular networks [J]. IEEE Transactions on Communications, Vol. 61(8) (2013), p. 3541-3551.

[14] K. Vanganuru, S. Ferrante, G. Sternberg. System capacity and coverage of a cellular network with D2D mobile relays, Military Communications Conference. (2012), p. 1-6.

[15] D. Lee, S. Kim, J. Lee, J. Heo. Performance of multihop decode-and-forward relaying assisted device-to- device communication underlaying cellular networks, International Symposium on Information Theory and its Applications. (2012), p. 455-459.

[16] D. Niyato, L. Xiao, P. Wang. Machine-to-machine communications for home energy management system in smart grid. IEEE Communications Magazine, Vol. 49(4) (2011), p.:53-59. 